\title{
The effect of lipiodol deposition in HCC after TACE on the necrosis range of PMCT
}

This article was published in the following Dove Press journal:

OncoTargets and Therapy

I August 2017

Number of times this article has been viewed

\author{
HongLiang Sun ${ }^{1,2}$ \\ JiaYan $\mathrm{Ni}^{1}$ \\ XiongYing Jiang' \\ Dong Chen' \\ YaoTing Chen' \\ LinFeng $\mathrm{Xu}^{\prime}$
}

'Department of Interventional Therapy, Sun Yat-Sen Memorial Hospital, Sun Yat-Sen University, Guangzhou, Guangdong Province, China; ${ }^{2}$ Department of Radiology, Children's Hospital of Philadelphia, Perelman School of Medicine, University of Pennsylvania,

Philadelphia, PA, USA
Correspondence: LinFeng Xu Department of Interventional Therapy, Sun Yat-Sen Memorial Hospital, Sun Yat-Sen University, Guangzhou 510120, Guangdong Province, China Tel +86 I38 26263989 Email xulinfeng_64@I26.com
Objective: To study the impact of lipiodol deposition in the lesion of hepatocellular carcinoma (HCC) after transarterial chemoembolization (TACE) on the necrosis area of percutaneous microwave coagulation therapy (PMCT).

Materials and methods: A total of 44 patients with $\mathrm{HCC}$ with 56 nodules, with a size ranging from 1.5 to $3.5 \mathrm{~cm}$, was selected in our study. About 23 patients ( 26 nodules) underwent PMCT treatment only as Group A and 21 patients (30 nodules) were treated by PMCT-combined TACE as Group B. All patients underwent PMCT with single-electrode and one-point ablation. Paired $t$-test was used to analyze pre- and postoperatively the volume of tumor and the necrosis volume after PMCT. Independent $t$-test was used to compare the difference in the necrosis area between two groups $(\alpha=0.05)$.

Results: All patients underwent PMCT or PMCT combined with TACE successfully. The tumor and necrosis size of Group A was $16.29 \pm 19.23 \mathrm{~cm}^{3}$ and $17.98 \pm 18.49 \mathrm{~cm}^{3}(P=0.650)$, and $11.95 \pm 12.78 \mathrm{~cm}^{3}$ and $16.60 \pm 11.70 \mathrm{~cm}^{3}$ of Group B $(P=0.017)$. There was no significant difference on necrosis volume between the two groups $(P=0.581)$. The necrosis area of Group $\mathrm{B}$ was larger than the size of the tumor $(P=0.017)$, but the ablation area of the two groups was smaller than the theoretic area $(P=0.001)$. (The theoretic area means that the necrosis area of ablation should be $1.0 \mathrm{~cm}$ larger than the tumor in diameter.)

Conclusion: PMCT combined with TACE could enlarge the ablation area, but will not lead to an ideal necrosis area than the PMCT alone. The lipiodol deposition in the tumor lesion may hinder the expansion of the heating field. Therefore, further research was needed.

Keywords: hepatocellular carcinoma, transarterial chemoembolization, percutaneous microwave coagulation therapy, lipiodol

\section{Background}

Hepatocellular carcinoma (HCC) is a common malignance throughout the world with a high morbidity and mortality rate. Although modern imaging techniques make the diagnosis of HCC much easier and accurate than before, the effect and prognosis is still not satisfactory.

Transarterial chemoembolization (TACE) is a combination of local delivery of chemical medicine and embolization procedure to cure solid tumor, mostly used for HCC. The use of TACE improves the prognosis of HCC, but the medium to long-term efficacy is not good enough.

Percutaneous microwave coagulation therapy (PMCT) is a micro-invasive technique by transferring microwave into the tumor lesion to generate heat, which leads to a complete necrosis of the HCC. But the rich blood supply around the tumor limited the spreading of the PMCT in the necrosis area. TACE has the advantage of reducing the local blood supply of the tumor, resulting in tissue necrosis and 
inflammatory edema. Theoretically, PMCT combined with TACE can reduce the "heat sinking" effect of blood flow during ablation to enlarge the coagulation necrosis of PMCT.

The institutional review board (IRB) of Sun Yat-Sen Memorial Hospital approved this study. Written informed consent was not required by the IRB as this was a retrospective review of anonymized patient data.

\section{Materials and methods}

This retrospective study including 44 patients with $56 \mathrm{HCC}$ nodules was selected from March 2011 to December 2014. Diagnosis was based on the CT or MRI imaging, alphafetoprotein (AFP) level, laboratory tests, or histology. All clinical data were obtained from the medical records and CT, and MRI images were collected from hospital PACS (picture archiving and communication systems).

\section{Clinical materials}

Forty-four patients (38 males and 6 females) with 56 tumor nodules of the $\mathrm{HCC}$, with size ranging from 1.5 to $3.5 \mathrm{~cm}$ in diameter, were enrolled in our study. The patients were divided into two groups according to the treatment. Group A with 26 nodules of 23 patients (58.04 \pm 12.93 years old) underwent PMCT only, while Group B with 30 nodules of 21 patients (51.71 \pm 14.74 years old) underwent both PMCT and TACE. The size of the tumor (diameter of three mutual perpendicular directions) of Group A was $2.99 \pm 1.50 \mathrm{~cm}$, $2.69 \pm 1.25 \mathrm{~cm}$, and $2.48 \pm 1.13 \mathrm{~cm}$, and the mean tumor volume was $16.29 \pm 19.23 \mathrm{~cm}^{3}$. For Group B, the tumor was $2.57 \pm 0.90 \mathrm{~cm}, 2.57 \pm 1.06 \mathrm{~cm}$, and $2.44 \pm 1.16 \mathrm{~cm}$ in diameter, and the mean volume of tumor was $11.95 \pm 12.78 \mathrm{~cm}^{3}$. Blood routine, coagulation, biochemical, biomarkers, and evaluation of the hepatic function was checked before operation (Child-Pugh standard). There was no significant difference between two groups on patients' age, tumor size, and hepatic function levels.

All patients were informed about the indication of therapy, the risk of interventional operation, recurrence rate, operation procedure, and postoperative treatment.

\section{Equipment}

Siemens 64-slice spiral CT machine (Somatom 64 Sensation, Munich, Germany) was used for image acquisition and PMCT puncture guidance Philips (INTERGRIS V3000 and ALLURE FD20) digital subtraction angiography (DSA) was used for interventional therapy of TACE. Routine apparatus included puncture needle, artery catheter sheath, catheter and micro catheter, lipiodol, and chemotherapeutics such as
5-FU and floxuridine, theraruibcin, hydroxycamptothecin, and mitomycin c.

The apparatus for microwave ablation was ECO-100 water-cooled microwave apparatus (Qinghai Electric Manufacturing, Nanjing, China) and monopole microwave antenna (18G).

\section{Patient selection criteria}

1. The selected patient with HCC or TACE treated failure

2. Nodule size from 1.5 to $3.5 \mathrm{~cm}$

3. Liver function was Child-Pugh class A or B

4. Suitable for PMCT treatment with single-electrode and single point ablation

5. Tumor with rich blood supply, but cannot be treated with TACE alone

6. Those without serious coagulation disorders

7. Patients agreeing to receive PMCT- or PMCT-combined TACE treatment.

\section{Therapeutic methods TACE}

Preoperative preparation

The patient was fasted for 12 hours before operation, both side of inguinal region skin prepared, and antibiotic and antacid drugs (omeprazole $40 \mathrm{mg}$ ) were given 30 minutes before the operation.

\section{Procedure of TACE}

The patient laid on back, after local anesthesia with $1 \%$ lidocaine at point $2 \mathrm{~cm}$ lower the middle of inguinal, Seldinger skill was used to puncture the right femoral artery. After the placement of the arterial sheath, 5F Yashiro or RH catheter was inserted. Then, the celiac artery and superior mesenteric artery angiography was done under the guidance of DSA to ascertain the blood supply in the liver and the tumor. In order to protect the function of normal hepatic parenchyma, superselective catheterization was done using a micro catheter (2.7F/2.8F Progreat, Terumo). Chemical medicine and lipiodol emulsion was injected through the catheter in the target artery. The dose of lipiodol used for TACE was 1-6 mL. The standard of complete embolization was that the whole tumor was filled with lipiodol emulsion, the blood flow was stopped, and the small portal vein around the tumor was filled with lipiodol. The sheath and catheter was pulled out after the operation.

\section{Postoperative management}

The vital signs of patients were monitored for 6-8 hours after the operation. The medicine for protecting hepatic function, 
preventing infection, pain killer and dealing with symptoms should be given at the same time. Blood routine and the levels of biochemical and biomarkers were retested 7 days later. The next treatment of PMCT was proposed to be given if there was no contradiction in Group B.

\section{PMCT}

Preoperative preparation

Absolute diet for 4-6 hours and transdermal fentanyl (4.2 mg) was given for analgesia 6 hours before PMCT operation. Antacid drug (omeprazole $40 \mathrm{mg}$, intravenously) and atropine $(0.5 \mathrm{mg}$, intramuscularly [IM]) was given 30 minutes before the procedure to inhibit gastric acid secretion and vagal reflex during the operation. Antibiotics also needed to be used prophylactically.

\section{Procedure of PMCT}

Conventionally, the patient lay supine with a fine metal marker on the body surface over the tumor. If the tumor was located in segment I or VII of the liver, in order to get a perfect path for puncture or to avoid damage to important vessels or normal organs, the patient had to lie prostrate or on the left side. To determine the pathway of puncture, a plain and enhancement CT scan image should be evaluated. The shortest distance with minimum damage, no big blood vessel and bile duct on pathway and easy to cooperate with the patient, were the principles of puncture pathway selection. After local anesthesia with $2 \%$ lidocaine at the puncture point, a $20 \mathrm{G}$ needle was punctured along the pathway as a locating needle. To confirm the puncture pathway, a plain $\mathrm{CT}$ scan was done. Once the pathway was determined, the microwave probe was inserted along the locating needle through the center of the tumor to its opposite margin. Before the microwave is switched on, $60-80 \mathrm{mg}$ dolantin should be injected IM for analgesia. And if the patient still cannot suffer the pain of ablation, general anesthesia had to be done. The vital signs were monitored during the operation. The probe and locating needle was pulled out after the ablation, and the wound of the skin was wrapped up. Plain CT scan again was the last step to find out whether there was a serious complication, such as the bleeding of abdominal or thoracic cavities or a massive pneumothorax. The $\mathrm{CT}$ image showed that the lesion density was lesser, while the tissue close to the electrode was a little higher, indicating the possibility of small bubbles in the lesion.

\section{Postoperative management}

After PMCT treatment, conventional treatment was given just like after TACE. The vital signs were monitored for
12 hours after the operation. Patients with complication of pneumothorax should be prolonged at the time of lying in bed until the air in thoracic cavity is absorbed, and thoracic drainage should be done if necessary. The liver and kidney function and the level of biomarkers were reexamined 7 days later and the patients discharged from hospital for recovery until their hepatic function returned to normal.

Periodic examination was needed for each patient. The first subsequent visit was 4-6 weeks later for laboratory tests, for assessing the level of tumor biomarkers, and for imaging evaluation (CT or MRI), and then the follow-up visit should be every 2 months for 3 times, and then once every 3 months. The longest follow-up time was 70 months with a mean of 24.5 months.

\section{Measurement and calculation}

Three mutual vertical diameters of the tumor and necrosis area of PMCT were measured using Siemens, Syngo Software Package on CT imaging, and among them, the long axis (a) was measured along the pathway of puncture.

The volume of tumor and necrosis area made by PMCT was calculated using the following formula:

$$
V=\frac{\pi}{6} \times a \times b \times c
$$

( $V$-volume, $a, b, c$ was three diameters of the tumor or necrosis, length $(a)$, width $(b)$, and height $(c))$.

\section{Statistics}

SPSS software package (version 16.0, SPSS Inc. Chicago, IL, USA) was used for statistical analysis. Paired-samples $t$-test was done to compare the parameters, such as blood routine, hepatic function, coagulation function, biomarkers level, and the size of necrosis and tumor, pre- and postoperation. Independent-samples $t$-test was used for ascertaining the size of the necrosis between two groups. Average values were expressed as mean \pm SD. $P$-value $<0.05$ was considered to be significant.

\section{Results \\ Common condition}

Group A consisted of 23 patients with 26 lesions, while Group B had 21 patients with 30 lesions (Table 1A). The tumor size of Group A was $16.29 \pm 19.23 \mathrm{~cm}^{3}$ and $11.95 \pm 12.78 \mathrm{~cm}^{3}$ for Group B, which showed no significant difference between two groups $(P=0.319$, Table 1B). The mean age of patients in Group A was 58.04 \pm 12.93 years old with 21 males and 2 females, while it was $51.71 \pm 14.74$ years 
Table IA Common condition of 2 groups ( $\mathrm{n}$, years)

\begin{tabular}{lll}
\hline & Group A & Group B \\
\hline Total number & 23 & 21 \\
Male & 21 & 17 \\
Female & 2 & 4 \\
Age, years & $58.04 \pm 12.93$ & $51.71 \pm 14.74$ \\
Total of nodules & 26 & 30 \\
Location of nodule & & \\
$\quad$ Left lobe & 4 & 5 \\
Right lobe & 22 & 25 \\
\hline
\end{tabular}

old for Group B with 17 males and 4 females. There was no significant difference on ages between two groups.

All patients were successfully managed with PMCT and TACE, without any fatal postoperative complication and mortality. The dose of lipiodol used for patients in Group B was $1.0-6.0 \mathrm{~mL}$.

\section{Laboratory test}

Blood routine, hepatic function, coagulation, and biomarkers were tested before the operation to evaluate whether patients could withstand TACE and PMCT. Seven days after operation, all items should be tested repeatedly to compare with those of preoperation.

White blood cell and platelets count returned to normal level 7 days after PMCT, but they were significantly lower than those of preoperative.

Aspartate aminotransferase, alanine aminotransferase and total bilirubin (T-BIL) levels rose slightly for the two groups, but there was no significant difference. Seven days later, they returned to normal and jaundice declined. The hepatic function pre- and postoperation within each group had a significant difference (paired-samples $t$-test), but there was no significant difference between two groups (Table 2).

The postoperation coagulation function of both groups declined mildly, but was still in normal range (Table 3).

The level of AFP and carbohydrate antigen $125\left(\mathrm{CA}_{125}\right)$, which were the two tumor biomarkers mainly surveyed, had no significant difference pre- and postoperation intergroup. One week after treatment, these two biomarkers declined nearly to half level of preoperation (Group A $P_{\mathrm{AFP}}=0.012$, Group B $P_{\mathrm{AFP}}=0.001$, Table 4).

\section{Tumor size and necrosis volume of PMCT}

The tumor size of two groups was shown in Table 1B. In Group A, the necrosis area of PMCT was a little larger than tumor size 1 month after treatment, but without significant difference $(P=0.650)$, while the volume of necrosis was significantly larger in Group $\mathrm{B}(P=0.017)$. But, a comparison of the necrosis volume between two groups showed no significant difference $(P=0.581)$ (Tables 5 and 6) (Figures 1-3).

The ideal necrosis area was that the diameter of ablation should be $1.0 \mathrm{~cm}$ larger than that of tumor at least, while, compared to this volume, the size of the necrosis area of two groups was significantly small ( $P=0.001$, respectively) (Table 7).

\section{Complications}

\section{TACE-related complications}

Hemorrhage, subcutaneous hematoma at puncture site, dissecting aneurysm, and pseudoaneurysm were common complications of TACE. However, most of them did not require treatment. In our study, none of the patients had subcutaneous hematoma. Embolization syndrome including nausea, vomiting, fever, local pain, and abdominal distension occurred most commonly, and lasted for 3-7 days. A total of 18 patients in Group B had more than one of these symptoms, which relieved 3-7 days after TACE. Ectopic embolization of adjacent organ such as gall bladder was mainly because the catheter did not reach the target vessel or the reflux of lipiodol emulsion, which did not appear in Group B.

\section{PMCT-related complications}

Common complications caused by PMCT include puncture site or hepatic hemorrhage, pain, soft tissue burn, pneumothorax, hemothorax, serious arrhythmia, peritonitis, liver abscess, and metastasis along needle tract, etc.

Totally, there were 56 PMCT operations in two groups. Discomfort and pain caused by heating were the most common complications during ablation, shown in 22 patients, of which six underwent general anesthesia and the rest endured the ablation after analgesic therapy. One case of pneumothorax occurred in each group. There was one case of pneumothorax occurred in each group, and the pneumothorax volume

Table IB Tumor size of 2 groups $\left(\mathrm{cm}, \mathrm{cm}^{3}\right)$

\begin{tabular}{lllll}
\hline & Diameter $\boldsymbol{a}$ & Diameter $\boldsymbol{b}$ & Diameter $\mathbf{c}$ & Tumor volume $\left(\mathbf{c m}^{3}\right)$ \\
\hline Group A & $2.99 \pm 1.50$ & $2.69 \pm 1.25$ & $2.48 \pm 1.13$ & $16.29 \pm 19.23$ \\
Group B & $2.57 \pm 0.90$ & $2.57 \pm 1.06$ & $2.44 \pm 1.16$ & $11.95 \pm 12.78$ \\
$t$ & 1.288 & 0.385 & 0.144 & 1.007 \\
$P$-value & 0.203 & 0.702 & 0.886 & 0.319 \\
\hline
\end{tabular}

Notes: Data presented as mean \pm SD. $a, b$ and $c$ were diameters of the tumor, length $(a)$, width $(b)$, and height (c). 
Table 2 Hepatic function changes pre- and 7 days postoperation (paired-samples t-test)

\begin{tabular}{cllll}
\hline & AST (U/L) & ALT $(\mathbf{U} / L)$ & T-BIL $(\mu \mathrm{mol} / \mathbf{L})$ & Alb $(\mathrm{g} / \mathbf{L})$ \\
\hline Group A & & & & \\
Preop & $63.27 \pm 67.42$ & $61.31 \pm 55.00$ & $27.48 \pm 30.56$ & $35.76 \pm 9.40$ \\
Postop & $49.35 \pm 52.47$ & $43.85 \pm 36.99$ & $19.55 \pm 12.52$ & $33.57 \pm 8.74$ \\
$t$ & 2.718 & 3.392 & 1.913 & 3.423 \\
P-value & 0.012 & 0.002 & 0.067 & 0.002 \\
Group B & & & & \\
Preop & $59.87 \pm 38.46$ & $60.80 \pm 71.66$ & $18.93 \pm 18.58$ & $38.38 \pm 6.02$ \\
Postop & $40.70 \pm 24.06$ & $32.37 \pm 27.89$ & $16.33 \pm 14.17$ & $36.09 \pm 5.61$ \\
$t$ & 4.278 & 2.912 & 1.227 & 4.395 \\
P-value & 0.000 & 0.007 & 0.230 & 0.000 \\
\hline
\end{tabular}

Note: Data presented as mean \pm SD unless otherwise specified.

Abbreviations: ALB, albumin; ALT, alanine aminotransferase; AST, aspartate aminotransferase; T-BIL, total bilirubin; Preop, preoperation; Postop, postoperation.

of two cases was $<10 \%$ of volume thoracic cavity which did not need to be treated. Mild pleural effusion occurred in 5 patients, with 2 in Group A and 3 in Group B, requiring no treatment. There was no skin burn in all patients.

\section{Discussion}

The treatment of HCC was quite a challenge and required a multidisciplinary approach. ${ }^{1}$ Now, interventional therapy has become the first choice for unresectable HCC., ${ }^{2,3}$ Regional treatment for HCC, including TACE, PMCT, radiofrequency ablation, percutaneous ethanol injection, and laser-induced thermotherapy, were all promising methods for HCC treatment, especially for patients with cirrhosis, hepatic insufficiency, unresectable tumor, or multifocal tumors. ${ }^{4-8}$

\section{Characteristic of PMCT and TACE}

The principle of PMCT was to use the heat-sensitive tumor cells and heating effect of microwave to cure the tumor in the liver. The microwave probe could generate heat after its insertion into the center of the lesion, which induced the coagulation necrosis of the tumor tissue to kill the tumor

Table 3 Coagulation of 2 groups pre- and postoperation

\begin{tabular}{cllll}
\hline & PT (s) & INR & APTT (s) & D-D mier (g/L) \\
\hline Group A & & & & \\
Preop & $15.50 \pm 8.74$ & $1.04 \pm 0.15$ & $27.22 \pm 8.50$ & $257.92 \pm 395.57$ \\
Postop & $13.54 \pm 6.10$ & $0.95 \pm 0.14$ & $23.77 \pm 6.79$ & $225.50 \pm 294.26$ \\
$t$ & 1.930 & 3.771 & 2.776 & 1.329 \\
P-value & 0.065 & 0.001 & 0.010 & 0.196 \\
Group B & & & & \\
Preop & $12.84 \pm 3.07$ & $1.11 \pm 0.28$ & $29.46 \pm 6.85$ & $280.50 \pm 305.85$ \\
Postop & $11.77 \pm 2.17$ & $0.99 \pm 0.19$ & $27.48 \pm 5.91$ & $201.83 \pm 114.61$ \\
$t$ & 3.687 & 4.447 & 5.397 & 1.607 \\
$P$-value & 0.001 & 0.000 & 0.000 & 0.119 \\
\hline
\end{tabular}

Note: Data presented as mean \pm SD unless otherwise specified.

Abbreviations: Preop, preoperation; Postop, postoperation; PT, prothrombin time; INR, international normalized ratio; APTT, activated partial thromboplastin time; D-D, D-dimer.
Table 4 Biomarkers of two groups pre- and postoperation

\begin{tabular}{lll}
\hline & AFP $(\mathbf{n g} / \mathbf{m L})$ & CA $_{125}(\mathbf{U} / \mathbf{m L})$ \\
\hline Group A & & \\
Preop & $137.85 \pm 268.99$ & $74.25 \pm 160.97$ \\
Postop & $99.53 \pm 208.43$ & $44.34 \pm 105.10$ \\
$t$ & 2.723 & 1.317 \\
$P$-value & 0.012 & 0.200 \\
Group B & & \\
Preop & $163.91 \pm 173.82$ & $80.90 \pm 195.90$ \\
Postop & $90.10 \pm 166.06$ & $37.59 \pm 75.67$ \\
$t$ & 3.543 & 1.820 \\
P-value & 0.001 & 0.079 \\
\hline
\end{tabular}

Note: Data presented as mean \pm SD unless otherwise specified.

Abbreviations: AFP, alpha-fetoprotein; $\mathrm{CA}_{122}$, carbohydrate antigen 125; Preop, preoperation; Postop, postoperation.

mass completely. After the first report by Seki et al, ${ }^{9}$ many researchers and clinical doctors tried to use this method to cure small HCC. And this method also became an important option for HCC. But, the "heat sinking" effect limited the range of PMCT. ${ }^{10,11}$ And the conformality of the PMCT area was another limit.

The TACE that was widely used in the clinic for HCC was already over 30 years; it was a combination of local chemotherapy and embolization. It was a procedure that mixed chemotherapeutics drugs with lipiodol injected into the HCC through its blood supply artery, to kill or repress the tumor by blocking blood supply and keep releasing chemicals. ${ }^{12}$ A lot of clinical studies had shown that the TACE has the following characteristics: reducing the toxicity of the whole body, increasing local chemical concentration, producing few complications and having good short-term effect. Therefore, TACE has become one of the first choices for unresectable HCC especially for B stage (BCLC 2010) and is widely used all over the world. And the disadvantage of TACE is that tumor cells remain alive in the lesion, although it is filled with lipiodol completely. ${ }^{13}$

Table 5 Tumor size and necrosis volume of PMCT of 2 groups $\left(\mathrm{cm}, \mathrm{cm}^{3}\right)$

\begin{tabular}{cllll}
\hline & Diameter $\boldsymbol{a}$ & Diameter $\boldsymbol{b}$ & Diameter $\boldsymbol{c}$ & Volume $\left(\mathrm{cm}^{3}\right)$ \\
\hline Group A & & & & \\
Preop & $2.99 \pm 1.50$ & $2.69 \pm 1.25$ & $2.48 \pm 1.13$ & $16.29 \pm 19.23$ \\
Postop & $3.55 \pm 1.09$ & $2.89 \pm 0.86$ & $2.67 \pm 0.88$ & $17.98 \pm 18.49$ \\
$t$ & -2.844 & -1.156 & -0.938 & -0.460 \\
P-value & 0.009 & 0.259 & 0.357 & 0.650 \\
Group B & & & & \\
Preop & $2.57 \pm 0.90$ & $2.57 \pm 1.06$ & $2.44 \pm 1.16$ & $11.95 \pm 12.78$ \\
Postop & $3.29 \pm 0.83$ & $2.96 \pm 0.79$ & $2.85 \pm 0.89$ & $16.60 \pm 11.70$ \\
$t$ & -4.575 & -2.700 & -2.332 & -2.522 \\
P-value & 0.000 & 0.011 & 0.027 & 0.017 \\
\hline
\end{tabular}

Notes: Data presented as mean \pm SD unless otherwise specified. $a, b$ and $c$ were diameters of the tumor or necrosis, length (a), width (b), and height (c). Abbreviation: PMCT, percutaneous microwave coagulation therapy. 
Table 6 Necrosis range of 2 groups after PMCT $\left(\mathrm{cm}, \mathrm{cm}^{3}\right)$

\begin{tabular}{lllll}
\hline & Diameter $\boldsymbol{a}$ & Diameter $\boldsymbol{b}$ & Diameter $\boldsymbol{c}$ & Volume $\left(\mathrm{cm}^{3}\right)$ \\
\hline Group A & $3.55 \pm 1.09$ & $2.89 \pm 0.86$ & $2.67 \pm 0.88$ & $17.98 \pm 18.49$ \\
Group B & $3.29 \pm 0.83$ & $2.96 \pm 0.79$ & $2.85 \pm 0.89$ & $16.60 \pm 11.70$ \\
$t$ & 0.147 & -0.926 & -1.200 & -0.556 \\
$P$-value & 0.884 & 0.359 & 0.235 & 0.581 \\
\hline
\end{tabular}

Notes: Data presented as mean \pm SD unless otherwise specified. $a, b$ and $c$ were diameters of necrosis, length (a), width $(b)$, and height (c).

Abbreviation: PMCT, percutaneous microwave coagulation therapy.

\section{PMCT plus TACE}

PMCT could induce complete necrosis of tumor tissue in its heating range. But the effective heating field depended on the heat conduction and many factors of heat losing, which limited the spreading of the necrosis area. It was thought that blocking local blood flow was an effective method to enlarge the range of necrosis of PMCT. Theoretically, TACE might increase heat effect of PMCT by the following aspects. First, to reduce the "heat sinking" effect in two ways, lipiodol blocked artery blood supply of the tumor and a small drop of lipiodol could flow into small branches of portal vein through collateral circulation between hepatic artery and portal vein around the tumor to block the blood flow of the portal vein. ${ }^{14,15}$ Besides, TACE could cause ischemia and inflammatory edema of the tumor tissue, which increases intracellular and interstitial water content, which in turn improves the heat effect of microwave ablation. So, TACE might enhance the result of PMCT ablation.

Therefore, PMCT combined with TACE could damage not only the center part but also the hepatic parenchyma around the tumor, which could increase the efficiency of regional treatment to the level of nonsurgical resection.

According to the above analysis, researchers considered that PMCT after TACE could enlarge the coagulation necrosis range absolutely.

At early stage, in Seki et $\mathrm{al}^{9}$ reports they used several probes or ablation repeatedly to cure the tumor for the limitations of apparatus. It was reported that the liver tumor (17 from 18 cases) was totally necrosis and the necrosis area was $5 \mathrm{~mm}$ larger than tumor area on CT scan. Among them, 14 patients used 4 probes, 2 patients used 5 probes, and 1 used 6 probes. In 2009, Yang et a ${ }^{14}$ reported that 41 tumor nodules $(<3.0 \mathrm{~cm})$ underwent TACE-combined PMCT treatment. According to $\mathrm{CT}$ images and pathology, 34 nodules were totally necrosis after PMCT surrounding with 3-5 mm necrosis of liver tissue as safe margin, 7 nodules (17\%) were necrosis without the safe margin. And other researchers used new apparatus to enlarge the effect of PMCT. ${ }^{16,17}$ But, the necrosis area of one-point ablation with single probe was not indicated in detail.

Now, most researchers considered that the combination of the two methods could take advantage from each other. Angiography could find small lesions neglected by ultrasound, CT and even MRI; furthermore, the deposition of lipiodol after TACE could provide guidance for PMCT puncture. TACE reduced blood flow and heat-sinking effect around the tumor by blocking tumor artery and/or portal vein, which was benefit to ablate tumor tissue for PMCT. The PMCT could kill the tumor cells completely within its effective heating field, while TACE cannot. The microwave probe with water-cooled tip could enlarge its coagulation field in one-point ablation (from $1.6 \times 2.4 \mathrm{~cm}$ to $4.7 \times 5.2 \mathrm{~cm}$ ), to reduce tumor load and the number of TACE performers. ${ }^{17,18}$ The recommended ablation area of the apparatus that we used for PMCT was $4.5-5.0 \mathrm{~cm}$ in long-axis direction.

\section{Does TACE enlarge PMCT range?}

In our study, we observed that the ablation area of most completely filled with lipiodol lesions was not enlarged, and there was also no 5-mm lower density region surrounding the nodule as "safe margin" shown on CT-enhanced images as expected. Statistical analysis showed that as compared to
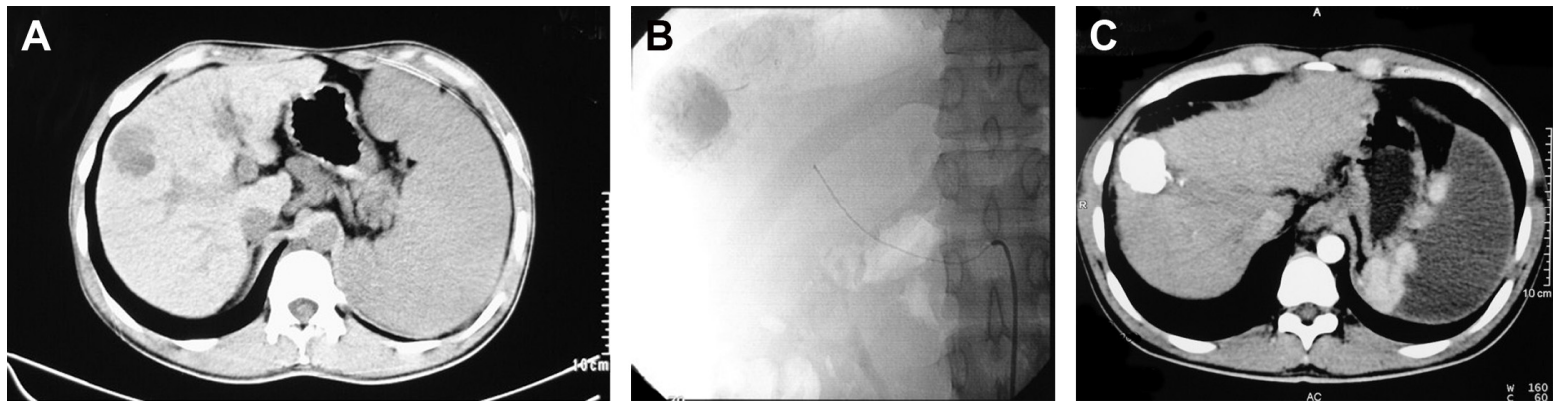

Figure I One male patient with HCC, the necrosis range of PMCT after TACE did not enlarged.

Notes: (A) Male patient with low-density lesion at segment VIII on plain CT scan (CT PS). (B) Lipiodol deposit well into the lesion after TACE, the same patient with (A). (C) One month after PMCT, lipiodol deposit well into the lesion, but no low-density belt surrounded the lesion.

Abbreviations: PMCT, percutaneous microwave coagulation therapy; TACE, transarterial chemoembolization. 


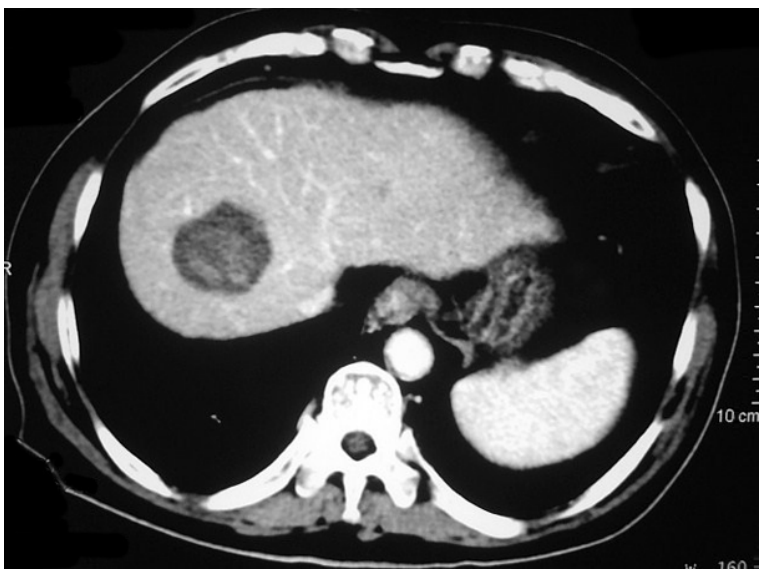

Figure 2 One month after PMCT only, the necrosis area surrounded the tumor area completely (CT CE).

Abbreviation: PMCT, percutaneous microwave coagulation therapy.

the PMCT group (necrosis area was $16.60 \pm 11.70 \mathrm{~cm}^{3}$ ), the necrosis area of the combination group $\left(17.98 \pm 18.49 \mathrm{~cm}^{3}\right)$ had no significant difference in size $(P=0.581)$. And the CT imaging showed that a different condition of lipiodol deposition of the Group B had an effect on the area and the shape of
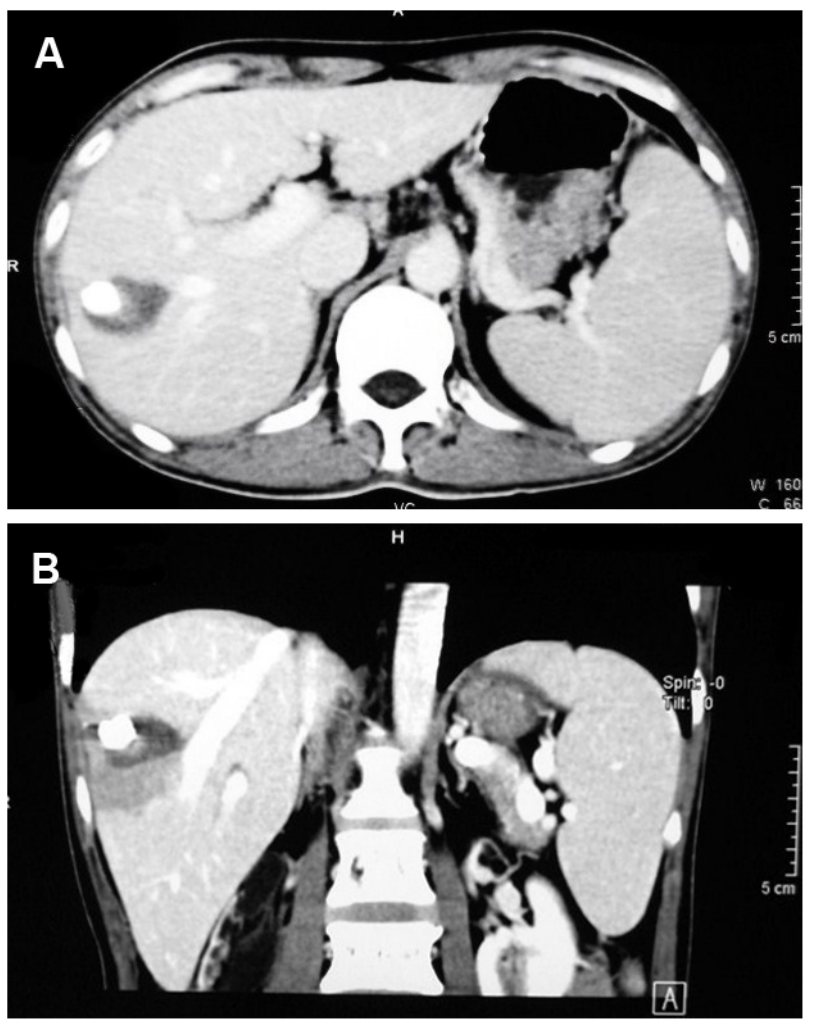

Figure 3 (A) One and a half months later, CT image showed that the heating area spread to the opposite direction of the surface liver because of the obstruction in the lipiodol deposition (CT CE axial). (B) One a and a half months later, the same case of $(\mathbf{A}), C T$ image showed that the heating area spread to the opposite direction of the liver surface (CT CE, coronal).
Table 7 Necrosis range of 2 groups after PMCT and ideal necrosis range $\left(\mathrm{cm}, \mathrm{cm}^{3}\right)$

\begin{tabular}{lllll}
\hline & Diameter $\boldsymbol{a}$ & Diameter $\boldsymbol{b}$ & Diameter $\mathbf{c}$ & Volume $\left(\mathrm{cm}^{3}\right)$ \\
\hline Group A & $3.55 \pm 1.09$ & $2.89 \pm 0.86$ & $2.67 \pm 0.88$ & $17.98 \pm 18.49$ \\
Ideal A & $3.99 \pm 1.46$ & $3.69 \pm 1.25$ & $3.48 \pm 1.13$ & $34.73 \pm 32.40$ \\
$t$ & -2.221 & -4.626 & -4.596 & -3.790 \\
P-value & 0.036 & 0.000 & 0.000 & 0.001 \\
Group B & $3.28 \pm 0.83$ & $2.96 \pm 0.79$ & $2.85 \pm 0.89$ & $16.60 \pm 11.70$ \\
Ideal B & $3.57 \pm 0.90$ & $3.57 \pm 1.06$ & $3.43 \pm 1.16$ & $27.82 \pm 22.53$ \\
$t$ & -1.838 & -4.165 & -3.310 & -3.555 \\
P-value & 0.076 & 0.000 & 0.003 & 0.001 \\
\hline
\end{tabular}

Notes: Data presented as mean \pm SD unless otherwise specified. $a, b$ and $c$ were diameters of necrosis, length (a), width (b), and height (c).

Abbreviation: PMCT, percutaneous microwave coagulation therapy

necrosis of the PMCT. If the lipiodol deposition fits the shape of tumor and filled completely, the necrosis area of the PMCT was rarely larger than the area of lipiodol deposition. But, if the lipiodol filled the tumor partially, the necrosis area of the PMCT would be usually larger than the tumor to form a "safe margin" with low density shown on CT imaging.

Why was the necrosis area of PMCT not enlarged after TACE? How many factors would have an effect?

Ionic conduction and dipole rotation are the main principles of microwave heating. So, the changes of the medium composition would affect the results of the microwave heat.

We reasoned that there may be several factors to count for this. First, the water content in the tumor nest was reduced after it was filled with lipiodol, which may reduce heat generation by PMCT. This phenomenon was shown obviously in patients with lipiodol completely deposition in tumor, meanwhile it was rare to observe the "safe margin" around the tumor on CT contrast enhancement images (Figures 1). Second, the heat generated by PMCT was limited to spread to the surrounding tissue because of low heat conduction of lipiodol. This may cause the distribution of heating field asymmetrical, which means that the necrosis was larger in lipiodol incompletely deposition area than that of completely deposition area (Figure 3A and B). Third, the timing for PMCT after TACE may have some effect. We did PMCT 7 days after TACE, after which the edema of tumor caused by TACE may have reduced.

Thus, as per our findings, we plan to use a different embolic agent and a different dosage of lipiodol in TACE before PMCT, and to conduct more animal experiments to improve the effect of combined treatment. ${ }^{19}$

\section{Conclusion}

PMCT could cause total necrosis of tumor in its heating field, and combine with TACE did not increase the occurrence 
of complications. But, remarkably, the coagulation field of combination treatment failed to achieve the theoretic necrosis area indicated that the deposition of lipiodol may limit the enlargement of the ablation area to some extent, which means more studies are needed to improve it.

\section{Disclosure}

The authors report no conflicts of interest in this work.

\section{References}

1. Bruera G, Cannita K, Giordano AV, et al. Multidisciplinary management of hepatocellular carcinoma in clinical practice. Biomed Res Int. 2014:806391.

2. Forner A, Reig ME, de Lope CR, Bruix J. Current strategy for staging and treatment: the BCLC update and future prospects. Semin Liver Dis. 2010;30(1):61-74.

3. Nishikawa H, Kimura T, Kita R, Osaki Y. Radiofrequency ablation for hepatocellular carcinoma. Int J Hyperthermia. 2013;29(6): 558-568.

4. Melia W, Dubbins P, Dubbins P, et al. Non-operative arterial embolization in primary liver tumors. Br Med J. 1979;2(6184):242-244.

5. Tateishi R, Shiina S, Teratani T, et al. Percutaneous radiofrequency ablation for hepatocellular carcinoma. An analysis of 1000 cases. Cancer. 2005;103(6):1201-1209.

6. Ohnishi K, Yoshioka H, Ito S, Fujiwara K. Prospective randomized controlled trial comparing percutaneous acetic acid injection and percutaneous ethanol injection for small hepatocellular carcinoma. Hepatology. 1998;27:67-72.

7. Ansari D, Andersson R. Radiofrequency ablation or percutaneous ethanol injection for the treatment of liver tumors. World J Gastroenterol. 2012;18(10):1003-1008.

8. Ohmoto K, Yoshioka N, Tomiyama Y, et al. Radiofrequency ablation versus percutaneous microwave coagulation therapy for small hepatocellular carcinomas: a retrospective comparative study. Hepatogastroenterology. 2007;54(76):985-989.
9. Seki T, Wakabayashi M, Nakagawa T, et al. Ultrasonically guided percutaneous microwave coagulation therapy for small hepatocellular carcinoma. Cancer. 1994;74(3):817-825.

10. Xu LF, Sun HL, Chen YT, et al. Large primary hepatocellular carcinoma: transarterial chemoembolization monotherapy versus combined transarterial chemoembolization-percutaneous microwave coagulation therapy. J Gastroenterol Hepatol. 2013;28(3):456-463.

11. Wakai T, Shirai Y, Suda T, et al. Long-term outcomes of hepatectomy versus percutaneous ablation for treatment of hepatocellular carcinoma $<$ or $=4 \mathrm{~cm}$. World J Gastroenterol. 2006;12(4):546-552.

12. Suzuki K, Kono N, Ono A, et al. Transcatheter arterial chemoembolization for humoral hypercalcemia of hepatocellular carcinoma. Gastroenterol Jpn. 1988;23(1):29-36.

13. Seki S, Sakaguchi H, Iwai S, et al. Five-year survival of patients with hepatocellular carcinoma treated with laparoscopic microwave coagulation therapy. Endoscopy. 2005;37(12):1220-1225.

14. Yang WZ, Jiang N, Huang N, et al. Combined therapy with transcatheter arterial chemoembolization and percutaneous microwave coagulation for small hepatocellular carcinoma. World J Gastroenterol. 2009;15(6):748-752.

15. Seki T, Tamai T, Nakagawa T, et al. Combination therapy with transcatheter arterial chemoembolization and percutaneous microwave coagulation therapy for hepatocellular carcinoma. Cancer. 2000; 89(6):1245-1251.

16. Yu Z, Liu W, Fan L, Shao J, Huang Y, Si X. The efficacy and safety of percutaneous microwave coagulation by a new microwave delivery system in large hepatocellular carcinomas: four case studies. Int $J$ Hyperthermia. 2009;25(5):392-398.

17. Inokuchi R, Seki T, Ikeda K, et al. Percutaneous microwave coagulation therapy for hepatocellular carcinoma: increased coagulation diameter using a new electrode and microwave generator. Oncol Rep. 2010;24(3):621-627.

18. Pan WD, Zheng RQ, Nan L, et al. Ultrasound-guided percutaneous microwave coagulation therapy with a "cooled-tip needle" for the treatment of hepatocellular carcinoma adjacent to the gallbladder. Dig Dis Sci. 2010;55(9):2664-2669.

19. Zhou P, Liu X, Li R, Nie W. Percutaneous coagulation therapy of hepatocellular carcinoma by combining microwave coagulation therapy and ethanol injection. Eur J Radiol. 2009;71(2):338-342.
OncoTargets and Therapy

\section{Publish your work in this journal}

OncoTargets and Therapy is an international, peer-reviewed, open access journal focusing on the pathological basis of all cancers, potential targets for therapy and treatment protocols employed to improve the management of cancer patients. The journal also focuses on the impact of management programs and new therapeutic agents and protocols on

\section{Dovepress}

patient perspectives such as quality of life, adherence and satisfaction. The manuscript management system is completely online and includes a very quick and fair peer-review system, which is all easy to use. Visit http://www.dovepress.com/testimonials.php to read real quotes from published authors. 